\title{
Functions of the Clostridium acetobutylicium FabF and FabZ proteins in unsaturated fatty acid biosynthesis
}

\author{
Lei Zhu ${ }^{\dagger 1}$, Juanli Cheng ${ }^{\dagger 1}$, Biao Luo ${ }^{1}$, Saixiang Feng1, Jinshui Lin ${ }^{1}$, \\ Shengbin Wang1, John E Cronan ${ }^{2,3}$ and Haihong Wang*1
}

Address: ${ }^{1}$ College of Life Science, South China Agricultural University, Guangzhou 510642, PR China, ${ }^{2}$ Department of Biochemistry, University of Illinois at Urbana-Champaign, Urbana, Illinois 61801, USA and ${ }^{3}$ Department of Microbiology, University of Illinois at Urbana-Champaign, Urbana, Illinois 61801, USA

Email: Lei Zhu - vermilionrock@yahoo.com; Juanli Cheng - chengjl-1981@163.com; Biao Luo - luobiao245@126.com; Saixiang Feng-211wsw@163.com; Jinshui Lin - linjinshui2@163.com; Shengbin Wang - swwl@scau.edu.cn; John E Cronan - jcronan@life.uiuc.edu; Haihong Wang* - wanghh36@scau.edu.cn

* Corresponding author †Equal contributors

Published: 4 June 2009

BMC Microbiology 2009, 9:119 doi:10.1186/1471-2180-9-119
Received: 29 January 2009

Accepted: 4 June 2009

This article is available from: http://www.biomedcentral.com/I47I-2/80/9/1/9

(C) 2009 Zhu et al; licensee BioMed Central Ltd.

This is an Open Access article distributed under the terms of the Creative Commons Attribution License (http://creativecommons.org/licenses/by/2.0), which permits unrestricted use, distribution, and reproduction in any medium, provided the original work is properly cited.

\begin{abstract}
Background: The original anaerobic unsaturated fatty acid biosynthesis pathway proposed by Goldfine and Bloch was based on in vivo labeling studies in Clostridium butyricum ATCC 6015 (now C. beijerinckii) but to date no dedicated unsaturated fatty acid biosynthetic enzyme has been identified in Clostridia. C. acetobutylicium synthesizes the same species of unsaturated fatty acids as $E$. coli, but lacks all of the known unsaturated fatty acid synthetic genes identified in $E$. coli and other bacteria. A possible explanation was that two enzymes of saturated fatty acid synthesis of $C$. acetobutylicium, FabZ and FabF might also function in the unsaturated arm of the pathway (a FabZ homologue is known to be an unsaturated fatty acid synthetic enzyme in enterococci).
\end{abstract}

Results: We report that the FabF homologue located within the fatty acid biosynthetic gene cluster of $C$. acetobutylicium functions in synthesis of both unsaturated fatty acids and saturated fatty acids. Expression of this protein in E. coli functionally replaced both the FabB and FabF proteins of the host in vivo and replaced $E$. coli FabB in a defined in vitro fatty acid synthesis system. In contrast the single $C$. acetobutylicium FabZ homologue, although able to functionally replace $E$. coli FabZ in vivo and in vitro, was unable to replace $\mathrm{FabA}$, the key dehydratase-isomerase of $E$. coli unsaturated fatty acid biosynthesis in vivo and lacked isomerase activity in vitro.

Conclusion: Thus, $C$. acetobutylicium introduces the double of unsaturated fatty acids by use of a novel and unknown enzyme.

\section{Background}

Bacterial growth requires an appreciable fraction of the acyl chains of the membrane lipids to be in a disordered state[1,2]. Such disordered states are imparted by fatty acids that act to offset the closely packed ordered arrangement of the lipid bilayer acyl chains imparted by straight- chain saturated acyl chains. In most bacteria the role of introducing acyl chain disorder is fulfilled by unsaturated fatty acids (UFAs). Some bacteria synthesize UFA by desaturation, an oxygen-requiring reaction that introduces the double bond in a single concerted reaction [2]. However, as first recognized by Bloch and coworkers this is not an 
option for anaerobically grown bacteria [3]. These investigators originally proposed that introduction of the double bond involved a direct dehydration of the 3-hydroxydecanoyl intermediate of fatty acid synthesis to give a cis-3 double bond which would be conserved though subsequent cycles of addition of two carbon atoms to give the membrane lipid UFA moieties [4]. However, when tested in cell-free extracts of $E$. coli, the reaction proved to proceed by a more conservative dehydration to give the classical trans-2-decenoyl fatty acid synthetic intermediate followed by isomerization of the trans-2-double bond to the cis-3 species $[3,5]$. This cis double bond was then preserved through successive $\mathrm{C}_{2}$ elongation cycles to form the double bond of the mature UFAs [6,7]. The dehydration and isomerization reactions were demonstrated by purification of the E. coli FabA enzyme (called the "Bloch dehydratase" to distinguish it from the E. coli FabZ dehydratase of the elongation cycle) that catalyzed both the dehydration and isomerization reactions(Fig. 1) [5]. Ironically, although the pathway was originally proposed based on the patterns of incorporation of short chain radioactive fatty acids into UFAs by cultures of Clostridium butyricum (now Clostridium beijerinckii) [4], all of the extant Clostridial genomes lack a homologue of FabA, the E. coli dehydratase-isomerase studied by Bloch and coworkers. Indeed, many bacterial genomes do not encode a recognizable FabA. This is also true of FabB, the E. coli chain elongation enzyme that channels the metabolic intermediate produced by FabA into the mainstream fatty acid synthetic pathway. Indeed in the extant genome

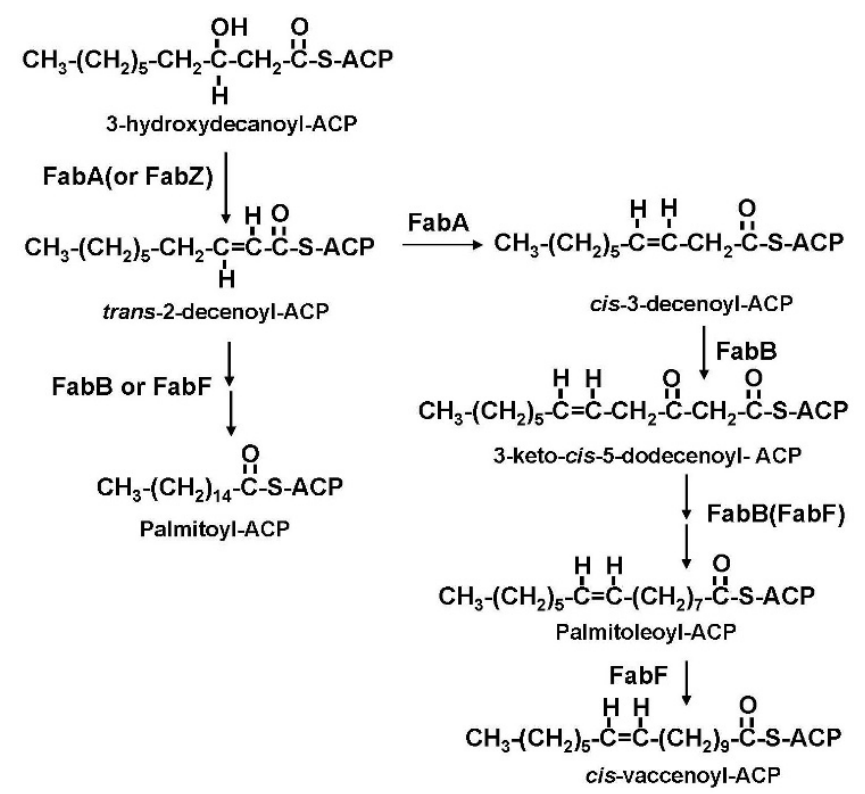

Figure I

Unsaturated fatty acid biosynthetic pathway of $E$. coli. sequences FabA and FabB homologues are encoded only in the genomes of $\alpha$ - and $\gamma$-proteobacteria $[6,7]$. Thus far, two solutions that solve the problem of anaerobic UFA synthesis in the absence of FabA and FabB have been reported. The first solution was that of Streptococcus pneumoniae which introduces a cis double bond into the growing acyl chain using FabM, a trans-2 to cis-3-decenoyl-ACP isomerase (i.e., the second partial reaction of FabA) [8]. The second solution was that of Enterococcus faecalis which uses homologues of FabZ and FabF to perform the functions performed by FabA and FabB in E. coli [9]. E. faecalis encodes two FabZ homologues and two FabF homologues (FabF is closely related to FabB). Wang and Cronan [9] showed that one of these proteins, now called FabN, functioned as a dehydratase/isomerase analogous to FabA, whereas the other FabZ homologue possessed only dehydratase activity. A similar picture was seen for the FabF proteins, one (now called FabO) performed the FabB function whereas the other functioned only as a FabF [9]. However, neither of these scenarios seemed applicable to the Clostridia. C. acetobutylicium lacks fabM, $f a b A$ and $f a b B$ and has only a single copy of $f a b Z$, although its fatty acid composition is similar to that of E. coli. This bacterium contains three genes that encode putative FabFs, although only one of these seemed likely to be involved in fatty acid synthesis (see Discussion). The most likely FabF homologue candidate was that encoded within a large gene cluster ( $f a b H a c p P f a b K, f a b D$ fabG $f a b F$ $a c c B f a b Z$ acc $C a c c D a c c A$ ) that encodes what appears to be a complete set of the genes required for saturated fatty acid synthesis. How does C. acetobutylicium make unsaturated fatty acids? One possibility was that the single FabZ and FabF homologues could somehow function in both the saturated and unsaturated branches of the fatty acid synthetic pathway. We report that the C. acetobutylicium FabZ cannot catalyze isomerization of its trans-2decenoyl-ACP product to the cis-3 species either in vitro or when expressed in E. coli. However, the single FabF homologue active in fatty acid synthesis has the functions of both E. coli long chain 3-ketoacyl-ACP synthases, FabB and FabF.

\section{Results \\ Only one of the three C. acetobutylicium fabF homologues can functionally replace $\mathrm{E}$. coli FabF in vivo} There are three annotated C. acetobutylicium fabF homologues designated as CAC3573, CAC2008 and CAA0093 $[10]$. We will temporarily call these genes $f a b F 1, f a b F 2$ and fabF3, although our data indicate that only the first of these genes functions in fatty acid synthesis. To test the functions of these homologues, the three genes were inserted into the arabinose-inducible vector pBAD24. The resulting plasmids were then introduced into two $E$. coli $f a b B(T s) f a b F$ strains, CY244 and JWC275. At the non-permissive temperature these mutant strains lack both long 
chain 3-ketoacyl-ACP synthase activities and thus are unable to grow even when the medium is supplemented with the unsaturated fatty acid, oleate $[11,12]$. Derivatives of strains CY244 or JWC275 carrying pHW36 encoding $\mathrm{fabF} 1$ grew at $42^{\circ} \mathrm{C}$ in the presence of oleate whereas the strains carrying pHW37 and pHW38 (encoding fabF2 and fabF3, respectively) failed to grow (Fig. 2) (similar results were seen with plasmids of both low and high copy number vectors). Thus, only fabF1 complemented the $E$. coli fabF mutation showing that $C$. acetobutylicium FabF1, like E. coli FabF, is able to catalyze all of the elongation reactions required in the synthesis of saturated fatty acids. Furthermore, expression of FabF1 restored thermal control of fatty acid composition to a FabF null mutant strain (Table 1). An E. coli fabF strain in which C. acetobutylicium FabF1 was expressed from the lac promoter of a low copy number vector closely mimicked the changes in fatty acid composition seen in wild type E. coli strains upon changes in growth temperature [13]. Expression of FabF1 restored cis-vaccenate synthesis at all temperatures, but was much more effective at $30^{\circ} \mathrm{C}$ than at $37^{\circ} \mathrm{C}$ or $42^{\circ} \mathrm{C}$ (Table 1 ). This effect seems likely to be due to the effects of temperature on FabF1 synthase activity since thermal regulation disappeared upon expression of FabF1 from a high copy number vector (Table 1) and the enzyme was thermolabile in vitro (see below). Apparently, at high growth temperatures low levels FabF1 elongation activity was overcome by high-level expression of the protein. We also found high levels of cis-vaccenate at the non-permissive temperature upon expression of $f a b F 1$ in an E. coli $f a b B$ $f a b F$ strain that carried the $f a b B$ gene of Haemophilus influenzae, a bacterium naturally defective in both cis-vaccenate synthesis and in regulation of fatty acid composition by temperature [14] (data not shown).

\section{The C. acetobutylicium fabF I gene can functionally replace E. coli FabB}

Although the presence of plasmid pHW36 (fabF1) allowed growth of the two E. coli $f a b B(T s) f a b F$ strains at the non-permissive temperature, growth of both strains required oleate. The lack of growth in the absence of oleate argued that either FabF1 lacked the ability to replace FabB or that FabF1 was unable to simultaneously perform the tasks of both FabB and FabF under these con- ditions. To decide between these alternatives we transformed pHW36 into strain K1060, a strain that carries an unconditional $f a b B$ allele, and into strain CY242 which carries the same $\mathrm{fabB}(\mathrm{Ts})$ allele as strain CY244. The complementation experiments showed that $C$. acetobutylicium fabF1 allowed strain K1060 to grow on RB medium lacking oleate at $37^{\circ} \mathrm{C}$ (Fig. 2). However, fabF1 failed to complement growth of the temperature sensitive $f a b B$ mutant strain, CY242 at $42^{\circ} \mathrm{C}$ (Fig. 2). If FabF1 possessed FabB activity at $37^{\circ} \mathrm{C}$, unsaturated fatty acids should be synthesized. To test this hypothesis, we grew strain K1060 carrying pHW36 or pHW33 (both encoding fabF1) at different temperatures and the fatty acid compositions of these strains were determined by collision induced dissociation electrospray mass spectroscopy (CID ES-MS) (Table 2). The strains clearly synthesized unsaturated fatty acids when grown at all of the different temperatures. However, the level of unsaturated fatty acids synthesized was lower than that seen in K1060 carrying a plasmid (pCY9) that encoded E. coli $f a b B$ and the amount of cis-vaccenate decreased with increased growth temperature. Moreover, despite the differing copy numbers, the two plasmids that encoded C. acetobutylicium FabF1 gave similar levels of unsaturated fatty acids. These results provide an explanation for lack of complementation of the $f a b B(T s)$ phenotype at $42^{\circ} \mathrm{C}$ by the $f a b F 1$-encoding plasmids. At $42^{\circ} \mathrm{C}$ the low activity of FabF1 did not allow enough unsaturated fatty acid synthesis to support growth. To test whether or not C. acetobutylicium FabF1 has FabB function at $42^{\circ} \mathrm{C}$ we assayed unsaturated fatty acid synthesis in strain CY242 carrying the fabF1 plasmid pHW36 (growth was supported by cyclopropane fatty acid supplementation) (Fig. $3)$. Under these conditions $\left[{ }^{14} \mathrm{C}\right]$ acetate labeling showed low levels of unsaturated fatty acids synthesis upon arabinose induction of FabF1 expression (Fig. 3). Therefore, FabF1 has the ability to replace FabB in E. coli unsaturated fatty acid synthesis but its expression allows growth only when the host FabF is present to perform the bulk of the chain elongation reactions.

\section{Functional analysis of C. acetobutylicium FabZ in vivo}

The sole fabZ homologue in the C. acetobutylicium genome is located within a large cluster of putative $f a b$ genes [10]. To test function of the encoded protein the $f a b Z$ gene was

Table I: Effects of growth temperature on fatty acid compositions (\% by weight)of fabF strain MR52 carrying plasmids encoding $C$. acetobutylicium fabFI.

\begin{tabular}{|c|c|c|c|c|c|c|}
\hline \multirow[b]{2}{*}{ Fatty acid } & \multicolumn{2}{|c|}{$30^{\circ} \mathrm{C}$} & \multicolumn{2}{|c|}{$37^{\circ} \mathrm{C}$} & \multicolumn{2}{|c|}{$42^{\circ} \mathrm{C}$} \\
\hline & pHW33 & pHW36 & pHW33 & pHW36 & pHW33 & $\mathrm{pHW} 36$ \\
\hline $\mathrm{Cl} 4: 0$ & 2.2 & 5.8 & 2.4 & 6.2 & 2.6 & 3.3 \\
\hline CI6:I & 40.3 & 29 & 35 & 24.8 & 53.4 & 28.9 \\
\hline $\mathrm{Cl} 6: 0$ & 21.4 & 25.8 & 32.4 & 25.1 & 26.2 & 28.7 \\
\hline CI8:I & 33.3 & 30 & 25.9 & 32.4 & 14.8 & 30.2 \\
\hline CI8:0 & 2.8 & 9.4 & 4.3 & 11.6 & 2.9 & 8.7 \\
\hline
\end{tabular}




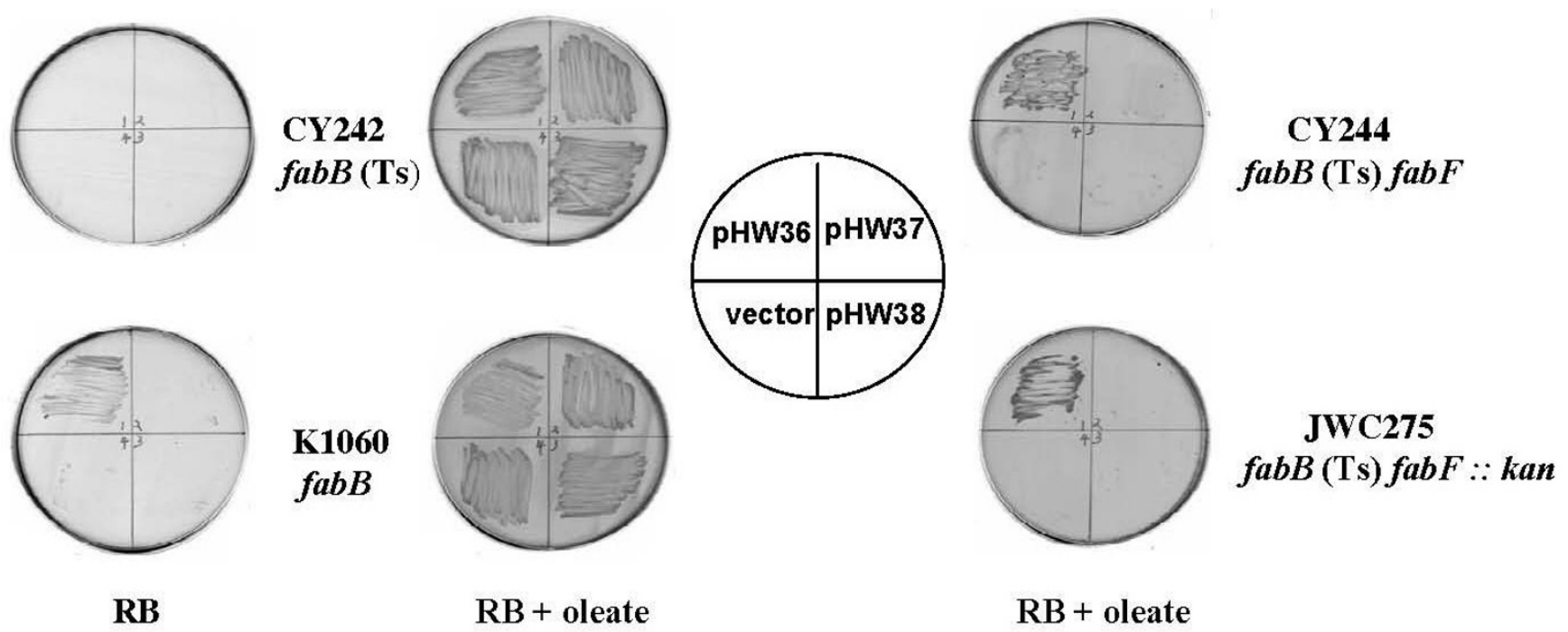

Figure 2

Growth of E. coli strains CY242, K1 060, CY244, and JWC275 transformed with plasmids encoding the C. acetobutylicium fabF homologues. Following induction by addition of arabinose, transformants of strain KI060 were grown at $37^{\circ} \mathrm{C}$, whereas the transformants of strains CY242, strain CY244 and strain JWC275 were grown at $42^{\circ} \mathrm{C}$. The strains carried plasmids pHW36, pHW37 or pHW38 encoding fabFI, fabF2 and fabF3, respectively, or the vector plasmid, pBAD24.

inserted into the arabinose-inducible pBAD24 expression vector to give plasmid pHW22. Since E. coli fabZ null strains are nonviable $[15,16]$, we first introduced pHW22 into strain DY330, a "recombineering" strain [17]. We then expressed the C. acetobutylicium FabZ in this strain and used standard phage $\gamma$ recombinase manipulations to delete the host fabZ gene. These manipulations gave strain HW7, which grew well in presence of arabinose but failed to grow in the presence of fucose, an anti-inducer of arabinose promoter expression (Fig. 4). The fatty acid composition of the complemented mutant strain grown in presence of arabinose was similar to that of the parental strain, DY330, indicating that C. acetobutylicium FabZ functionally replaced E. coli FabZ (Table 3). The lack of $f a b A$ and $f a b M$ homologues in C. acetobutylicium raised the possibility that the FabZ of this organism might function as both an isomerase and a dehydratase as does the E. faecalis FabZ-like protein, FabN [9]. To test this possibility plasmid pHW22 was introduced into both the fabA(Ts) $E$. coli strain CY57 and the fabA null mutant strain MH121. Neither stain grew in the absence of unsaturated fatty acid supplementation (data not shown) indicating that C. acetobutylicium FabZ lacks isomerase function and thus was unable to functionally replace FabA. However, it remained possible that $C$. acetobutylicium FabZ catalyzed UFA synthesis, but that the levels of UFA produced were too low to support growth. This possibility was tested by $\left[{ }^{14} \mathrm{C}\right]$-acetate labeling of the fatty acids synthesized by strain CY57 carrying pHW22 and analysis of the resulting radioactive fatty acids for traces of UFA (Fig. 5). No UFA synthesis was detected. Another possible explanation for the observed lack of UFA synthesis was that FabI, the enoyl-ACP reductase of $E$. coli, converted the intermediate trans-2-decenoyl-ACP to decanoyl-ACP before the putative isomerase activity of $C$. acetobutylicium FabZ could act. Thus, we repeated the labeling experiment in the presence

Table 2: Fatty acid compositions (\% by weight) of fabB strain K 1060 transformed with plasmids encoding either C. acetobutylicium fabFI or E. coli fabB.

\begin{tabular}{|c|c|c|c|c|c|c|c|c|c|}
\hline \multirow[b]{2}{*}{ Fatty acid } & \multicolumn{3}{|c|}{$30^{\circ} \mathrm{C}$} & \multicolumn{3}{|c|}{$37^{\circ} \mathrm{C}$} & \multicolumn{3}{|c|}{$42^{\circ} \mathrm{C}$} \\
\hline & pHW33 & pHW36 & $\mathrm{pCY} 9$ & pHW33 & pHW36 & $\mathrm{pCY} 9$ & pHW33 & pHW36 & $\mathrm{pCY} 9$ \\
\hline $\mathrm{Cl} 4: 0$ & 4.9 & 9.2 & 2.2 & II.I & 7.7 & 4 & II.I & 9.9 & 2.5 \\
\hline CI6:I & 12.8 & 8.1 & 16.8 & 17.5 & 18 & 20 & 19.7 & 13.5 & 20.3 \\
\hline $\mathrm{Cl} 6: 0$ & 22.1 & 21.6 & 10.8 & 25.9 & 23.6 & 13.8 & 32.6 & 42.7 & 19.7 \\
\hline C18:I & 43.1 & 43.1 & 67.1 & 31.8 & 34.4 & 58.1 & 17.7 & 22.4 & 51 \\
\hline C18:0 & 17 & 18 & 3.2 & 13.7 & 16.3 & 3.7 & 18.9 & 11.5 & 6.5 \\
\hline
\end{tabular}




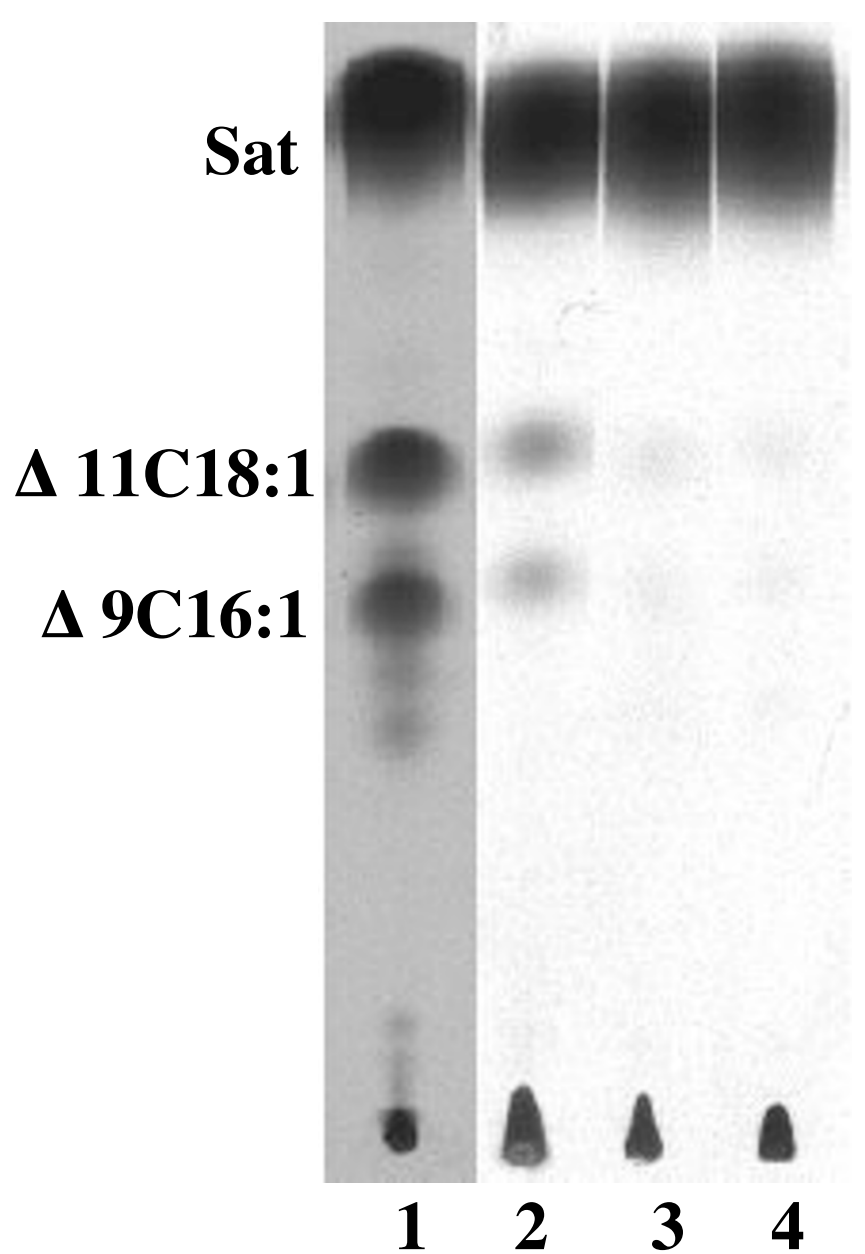

Figure 3

Expression of $C$. acetobutylicium FabF I restores UFA synthesis to $E$. coli fabB strains. The methyl esters of fatty acids were obtained from the phospholipids as described in Methods. Lane $I$ is the esters of the wild type $E$. coli strain MG I655. Lane 2 is the esters of strain CY242 carrying $\mathrm{PHW} 36$ (fabFl) in presence of arabinose induction. Lane 3 is the esters of strain CY242 carrying pHW36 (fabFI) in the absence of induction. Lane 4 is the esters of strain CY242 carrying vector PBAD24. The migration positions of the methyl esters of the fatty acid species are shown. The designations are: Sat, saturated fatty acid esterss; $\Delta 9 \mathrm{Cl} 6: \mathrm{I}$, methyl ester of cis-9-hexadecenoic; $\Delta \mathrm{IICI8:I,} \mathrm{methyl} \mathrm{ester}$ of cis-I I-octadecenoic.

of a low dose of triclosan, a specific E. coli FabI inhibitor [6], in order to give the putative isomerase a better opportunity to act on the trans-2-decenoyl-ACP intermediate. Again no synthesis of unsaturated fatty acid was observed (data not shown). These in vivo results argued strongly that that $C$. acetobutylicium FabZ was unable to isomerize trans-2-decenoyl-ACP.
Table 3: Composition of fatty acids of strain HW7

\begin{tabular}{lllll}
\hline & \multicolumn{4}{c}{ Fatty acid composition (\% by weight) } \\
\cline { 2 - 5 } & Cl4:0 & Cl6:I & Cl6:0 & Cl8:I \\
\hline DY330 & 3.2 & 41.0 & 29.7 & 26.0 \\
HW7 & $<0.5$ & 49.6 & 29.2 & 21.2 \\
\hline
\end{tabular}

\section{In vitro assay of $C$. acetobutylicium FabZ and FabFI activities}

To allow direct assay of C. acetobutylicium FabF1 and FabZ activities we expressed the proteins in E. coli to facilitate their purification. $\left[{ }^{35} \mathrm{~S}\right]$ Methionine labeling showed that strain BL21 (DE3) carrying plasmids encoding either $C$. acetobutylicium fabF1 or fabZ under control of a phage T7 promoter expressed proteins of the expected sizes (Fig. $6 \mathrm{~A})$. However, the expression level of the FabZ protein was so low that it was not detected upon staining the SDS gels (Fig. 6B). We attributed this poor expression to the fact that the $C$. acetobutylicium FabZ gene contains 24 codons that correspond to nonabundant (rare) tRNA species in E. coli. We therefore changed these codons to synonymous codons that correspond to abundant E. coli tRNA species thereby resulting in a modified gene we call fabZm. Plasmid pHW74m (which encoded the His-tagged fabZm under T7 promoter control) abundantly expressed a protein with an apparent mass of $17 \mathrm{kDa}$ (Fig. 6B) in good agreement with the expected value for the $\mathrm{His}_{6}$ tagged protein $(17.5 \mathrm{kDa})$. The $\mathrm{His}_{6}$-tagged FabZ protein

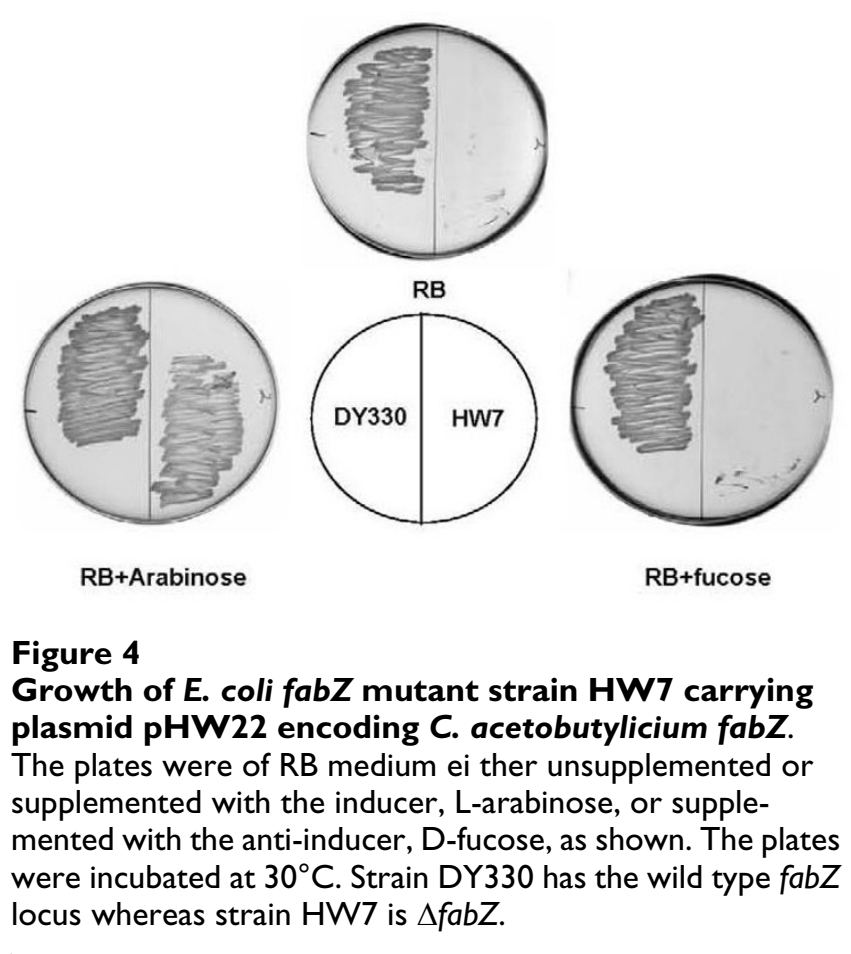




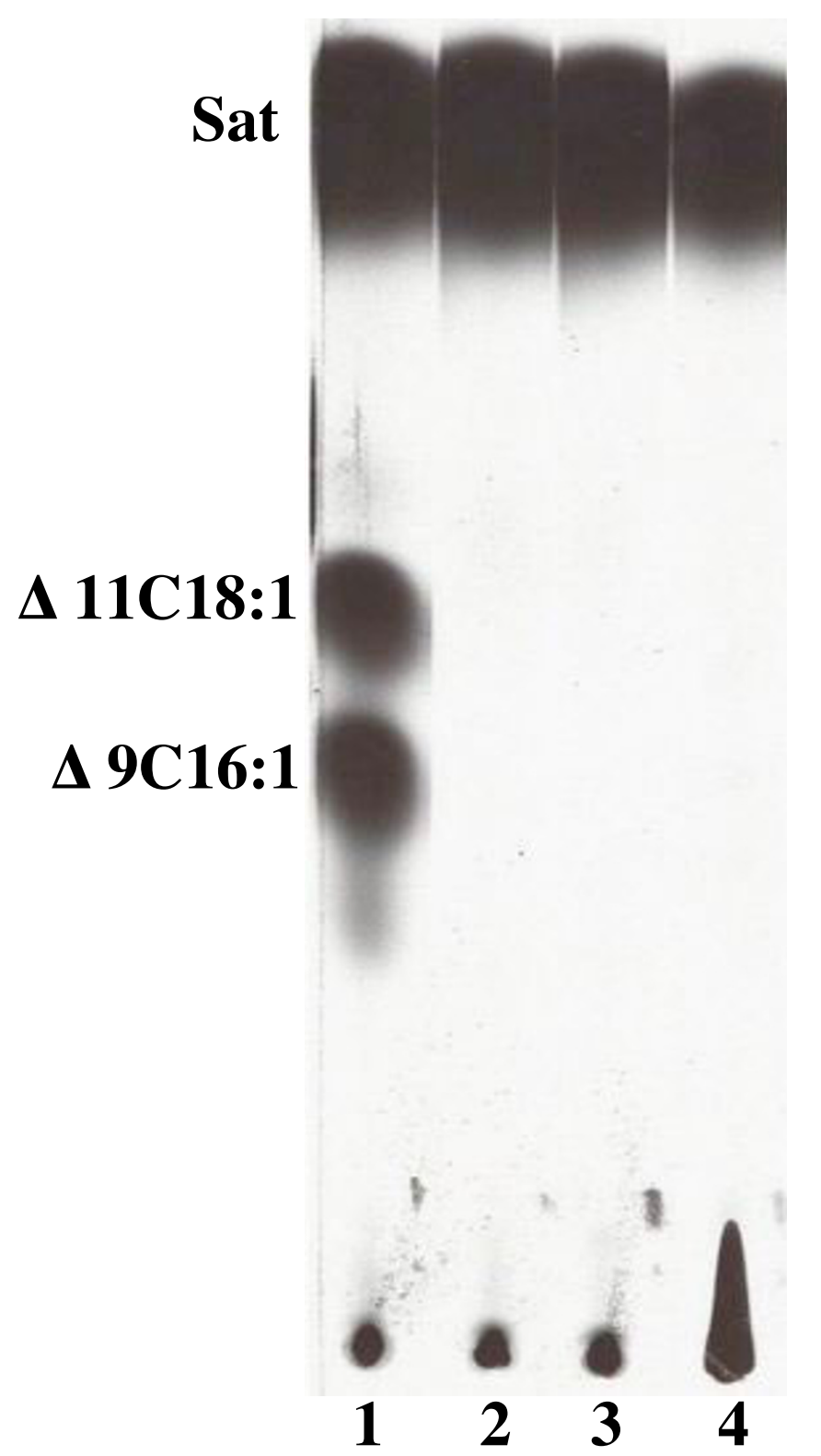

Figure 5

Argentation thin-layer chromatographic analysis of [ $\left.\mathrm{I}-{ }^{14} \mathrm{C}\right]$ acetate-labeled esters from strain CY57 transformed with pHW22 encoding $C$. acetobutylicium fabZ. The methyl esters of fatty acids were obtained from the phospholipids as described in Methods. Lane I is the methyl esters of the wild type $E$. coli strainMG I655. Lane 2 is the esters of strain CY57 carrying vector pBAD24. Lane 3 is the esters of strain CY57 carrying PHW22 which encodes the $C$. acetobutylicium fabZ labeled in the absence of induction. Lane 4 is the esters of strain CY57 (pHW22) following arabinose induction. Labels are as in Fig. 2.

was purified to essential homogeneity using nickelchelate chromatography (Fig. 6B). We also purified the Nterminally His $_{6}$-tagged versions of $C$. acetobutylicium FabF1 and the E. coli fatty acid biosynthetic proteins
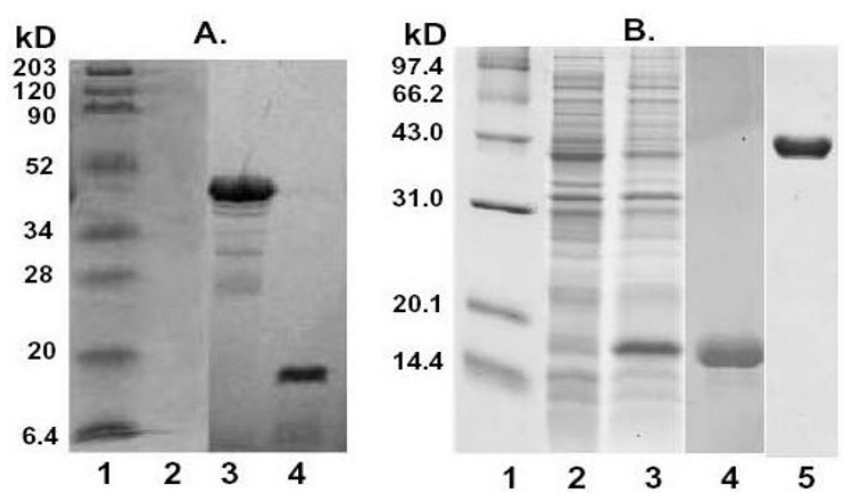

Figure 6

Expression of $C$. acetobutylicium FabZ and FabF I in $E$. coli. Panel A. Expression of C. acetobutylicium FabFI and FabZ from their native coding sequences was induced in $E$. coli BL2 I (DE3) under control of a phage T7 promoter. Lane: I, molecular mass markers; lane 2, proteins expressed in the presence of vector pET28b; lane 3, proteins expressed in the presence of $\mathrm{pHW} 28$ (FabFI) and lane 4, proteins expressed in the presence of pHW39 (FabZ). Panel B. An expression plasmid encoding the codon-optimized $C$. acetobutylicium fabZ was introduced into E. coli BL2I (DE3). Lane: I, molecular mass markers; lane 2, plasmid pHW74 which expresses native fabZ; lane 3, plasmid pHW74m which expresses the codon-optimized fabZ; lane 4, FabZ expressed from the codon-optimized gene purified by nickel-chelate chromatography and lane 5, FabFI purified by nickel-chelate chromatography.

FabD, FabG, FabA, FabZ, FabB and FabI plus the Vibrio harveyi AasS acyl-ACP synthetase [18] by nickel-chelate chromatography. AasS was used to synthesize the 3hydroxydecanoyl-ACP substrate whereas the other enzymes were used to assemble a defined in vitro fatty acid synthesis system in which the activities of E. coli FabA and C. acetobutylicium FabZ or E. coli FabB and C. acetobutylicium FabF1 could be directly compared. In reactions containing FabA 3-hydroxydecanoyl-ACP was converted to a mixture of trans-2 and cis-3-decenoyl-ACPs as expected from prior work $[19,20]$. E. coli FabB is unable to elongate trans-2-decenoyl-ACP, but elongates the cis-3 species to 3keto-cis-5-dodecenoyl-ACP in the presence of malonylACP [20]. This product is then reduced by FabG and dehydrated by FabA to form trans-2-cis-5-dodecadienoylACP[20]. The trans-2-cis-5-dodecadienoyl-ACP product accumulates because the reaction mixtures lacked enoylACP reductase which precluded further elongations [20]. Using this system we showed (as has long been known) that FabA is a bifunctional enzyme that catalyzes both the dehydration of 3-hydroxydecanoyl-ACP and well as the reversible isomerization of trans-2-decenoyl-ACP to cis-3decenoyl-ACP (Fig. 7). As expected E. coli FabZ converted 3-hydroxydecanoyl-ACP to trans-2-decenoyl-ACP. However, addition of $E$. coli FabB to this reaction failed to give the 12-carbon unsaturated elongation product seen with 
FabA (Fig. 7) in agreement with prior reports that E. coli FabZ acts solely as a dehydratase and that FabB is unable to elongate trans-2-decenoyl-ACP [20]. If C. acetobutylicium FabZ was capable of the isomerization reaction, then upon addition of $E$. coli FabB the reaction would yield trans-2, cis-5-dodecadienoyl-ACP [20]. However, the only product formed was trans-2-decenoyl-ACP, the product of E. coli FabZ (Fig. 7A). Hence, we conclude that C. acetobutylicium FabZ possesses only dehydratase activity and introduction of the cis double bond requires another enzyme that has yet to be discovered. In parallel experiments, we replaced E. coli FabB with $C$. acetobutylicium FabF1 in the E. coli FabA reaction mixture to test if C. acetobutylicium FabF1 could elongate cis-3-decenoyl-ACP (Fig. 7B). We found that addition of FabF1 gave a modest conversion of cis-3-decenoyl-ACP to trans-2-cis-5-dodecadienoyl-ACP and at $37^{\circ} \mathrm{C}$ the product yields were lower than those seen at $25^{\circ} \mathrm{C}$ and $30^{\circ} \mathrm{C}$ consistent with the low activity of FabF1 at high temperature seen in vivo (Fig 7B).

\section{Discussion}

Although C. acetobutylicium, C. beijerinckii and E. coli synthesize the same species of unsaturated fatty acids [21]

$\mathbf{A}$

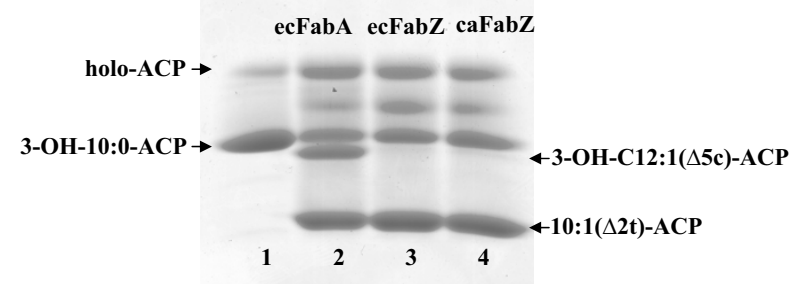

B

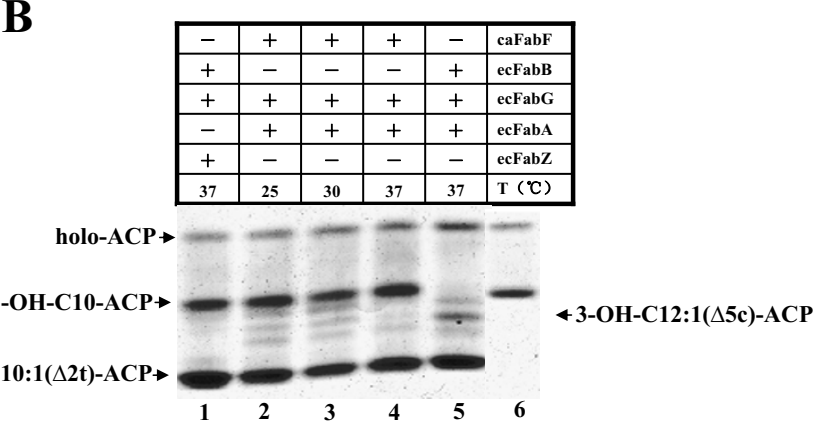

Figure 7

Properties of $C$. acetobutylicium FabZ and FabFI in vitro. Panel $A$. The ability of $C$. acetobutylicium FabZ to synthesize fatty acids was determined by conformationally-sensitive gel electrophoresis. Lanes: lane I, no addition; lane 2, E. coli FabA (ecFabA) was added; lane 3, E. coli FabZ (ecFabZ) was added and lane 4, $C$. acetobutylicium FabZ (caFabZ) was added. Panel $B$. The reactions shown above the gel were as in lane 2 except that $E$. coli FabB was replaced with $C$. acetobutylicium FabFI (caFabF) in lanes 2-4. Lane 6 is the 3-hydroxydecanoyl-ACP standard as in lane I of panel $A$. and Clostridia are thought to follow the same synthetic mechanism as E. coli [22], the enzyme that introduces the cis double bond of the unsaturated fatty acids remains unknown. Like other Clostridia the C.acetobutylicium genome encodes none of the three known anaerobic unsaturated fatty acid synthesis pathways denoted by the presence of genes encoding FabM, FabA or FabN proteins. One possibility was that the single FabZ of this bacterium could somehow partition acyl chains between the saturated and unsaturated branches of the pathway. However, our in vivo and in vitro data show that $C$. acetobutylicium FabZ cannot synthesize the first intermediate in unsaturated fatty acid synthesis. Hence, Clostridia must contain a novel enzyme that introduces the cis double bond. Note that the proposed isomerase activity of the C. acetobutylicium FabZ was not unreasonable. C. acetobutylicium FabZ shares 51.4 and $59.3 \%$ identical residues with E. faecalis FabN and FabZ, respectively, and there is no sequence signature that denotes isomerase ability $[9,23,24]$. This is because the isomerase potential of 3-hydroxyacyl-ACP dehydratases is not determined by the catalytic machinery at the active site but rather by the $\beta$-sheets that dictate the orientation of the central $\alpha$-helix and thus the shape of the substrate binding tunnel $[23,24]$. We are currently seeking the gene(s) that encode the enzyme responsible for cis double bond introduction in C. acetobutylicium.

In contrast to FabZ, the single 3-ketoacyl-ACP synthase (FabF) of this bacterium performs the elongation functions required in both branches of the fatty acid synthetic pathway. This protein can both elongate palmitoleoylACP to cis-vaccenoyl-ACP as does FabF in E. coli and also elongates the cis double bond containing product of FabA as does E. coli FabB. However, C. acetobutylicium FabF, was unable to perform the two tasks simultaneously and thus differs from Enterococcus faecalis FabO [9]. Although the C. acetobutylicium FabF and E. faecalis FabO proteins are 45$46 \%$ identical to E. coli FabF, they are only 55\% identical to one another. Hence, each of the three proteins is distinct from the other two. The finding that C. acetobutylicium FabF was unable to perform the two tasks simultaneously could be due to the intrinsic temperature sensitivity of FabF1 and to the enzyme undergoing a type of kinetic confusion in this unnatural setting. Perhaps the intermediates of one branch of the pathway act (in effect) as inhibitors of the other branch. In this scenario the presence of the E. coli enzyme (either FabB or FabF) would result in the inhibitory intermediates being converted to long chain acyl chains, thereby freeing the C. acetobutylicium FabF to operate in the other branch. The complex task faced by FabF1 upon expression in an E. coli strain lacking both FabB and FabF is illustrated by the effects of overproduction of FabA and FabB in E. coli [25]. Overproduction of FabA results in increased production of saturated fatty acids rather than the increase in unsaturated 
fatty acid levels that might have been expected [25]. In contrast overproduction of FabB has the opposite result; unsaturated fatty acid levels are increased [25]. However, if the two enzymes are simultaneously overproduced, the fatty acid composition returns to normal [25]. These counter-intuitive results are due to the fact that FabA catalyzes reversible reactions whereas the FabB reaction is irreversible. Hence, when FabB activity is limiting, any excess cis-3-decenoyl-ACP produced by FabA can be isomerized back to trans-2-decenoyl-ACP and upon FabI action, this acyl chain can enter the saturated arm of the pathway. However, when FabB is in excess, it catalyzes the irreversible elongation of cis-3-decenoyl-ACP and thereby pulls the flow of carbon toward the unsaturated branch of the pathway. Thus, it would seem a surprising finding if the $C$. acetobutylicium FabF was able to accurately partition acyl chains between the two branches of the fatty acid synthetic pathway of a foreign organism.

It should be noted that it was not unexpected that the FabF homologue encoded within the fab gene cluster was the only FabF homologue that functioned in fatty acid synthesis. There are good arguments against the other two homologues having this function. The CAC2008 ORF in located within a cluster of genes that appear involved in synthesis of a glycosylated product of a hybrid polyketidenonribosomal polypeptide pathway. If so, the CAC2008 ORF would be involved in synthesis of the polyketide moiety. The CAA0088 ORF is encoded on the C. acetobutylicium megaplasmid required for the late steps of solvent production by this organism. C. acetobutylicium survives loss of the megaplasmid [26] and therefore the CAA0088 ORF cannot encode an enzyme essential for fatty acid synthesis (although it could still provide FabF function). Note that it has been recently reported that the single FabF protein of the distantly related gram positive bacterium Lactococcus lactis can also perform the FabB reaction as well as that of FabF[27].

\section{Conclusion}

Unsaturated fatty acid synthesis in Clostridia cannot be explained by a plenipotent FabZ indicating that these bacteria encode a novel enzyme that introduces the cis double bond. In contrast the Clostridia FabF protein has the functions of both of the long chain 3-ketroacyl-ACP syntheases of $E$. coli. The diversity of bacterial enzymes used for synthesis of the cis double bond of unsaturated fatty acids is unexpected because the remainder of the fatty acid synthetic enzymes is well conserved among very diverse bacteria.

\section{Methods}

\section{Bacterial strains, plasmids and growth conditions}

The E. coli strains and plasmids used in this study are listed in Additional file 1. Luria-Bertani medium was used as the rich medium for $E$. coli. The phenotypes of $f a b$ strains were assessed on rich broth (RB) medium [12]. Oleate neutralized with $\mathrm{KOH}$ was added to RB medium at final concentration of $0.1 \%$ and solubilized by addition of Brij 58 detergent to final concentration of 0.1 to $0.2 \%$. Antibiotics were used at the following concentrations (in $\mathrm{mg} / \mathrm{L}$ ) sodium ampicillin, 100; chloramphenicol, 30; kanamycin sulfate and rifampicin, 200. L-Arabinose and Dfucose were used at concentrations of $0.01 \%$. Isopropyl- $\beta$ D-thiogalactoside (IPTG) was used at final concentration of $1 \mathrm{mM}$.

\section{Recombinant DNA techniques and construction of plasmids}

Restriction enzymes, T4 DNA ligase and Taq DNA polymerase were from Invitrogen or New England Biolabs unless indicated otherwise. All enzymatic reactions were carried out according to the manufacturer's specifications. Qiagen products were used to isolate plasmids, purify DNA fragments from agarose gels and purify PCR products. Plasmids were introduced into E. coli strains by $\mathrm{CaCl}_{2}$-mediated transformation. C. acetobutylicium ATCC824 genomic DNA was extracted using the GNOME DNA kit (Bio 101). DNA sequencing and the synthesis of oligonucleotides were done at the University of Illinois Keck Genomics Center.

The C. acetobutylicium fabF homologues were amplified from genomic DNA using the primers $f a b F 1, f a b F 2$ and fabF3 (Additional file 1). The PCR products were cloned into vector pCR2.1TOPO to give plasmids pHW40 (fabF1), pHW41 (fabF2) and pHW42 (fabF3). Plasmids pHW40 and pHW42 were then digested with EcoRI, the appropriate fragments were isolated and these were ligated into pHSG576 [28] digested with the same enzyme to give plasmids pHW33 and pHW35, respectively. The orientation of the C. acetobutylicium ORFs in these plasmids were such that the genes would be transcribed by the vector lac promoter. The HindIII-XhoI fragment of pHW41 was ligated into vector pSU20 [29] digested with the same enzymes to give pHW43 which was then digested with HindIII plus SalI and the fabF2containing fragment was inserted into the same sites of vector pHSG576 to give pHW34. Plasmids pHW16, pHW31 and pHW32 were constructed as follows. The upstream primers were primers12,34 and 56 (Additional file 1) and the downstream primer was the M13 (-) forward primer. Plasmids pHW33, pHW34 and pHW35 were used as templates for PCR amplification. The products were cloned into vector pCR2.1 TOPO to yield pHW16, pHW31 and pHW32, respectively. The BspHI-PstI fragments of pHW16 and pHW32 were then ligated into NcoI and PstI sites of pBAD24 [30] to give plasmids pHW36 and pHW38, respectively. Likewise, the BspHI-HindIII fragment of pHW31 was inserted into the NcoI and HindIII sites of pBAD24 to yield pHW37. 
The fabZ homologue was amplified by PCR using C. acetobutylicium genomic DNA as template with primers Zprimer1 and Zprimer2 (Additional file 1). The PCR product was inserted into pCR2.1 TOPO vector to give pHW15. The BspLU11I-HindIII fragment of pHW15 was inserted into the sites of pBAD24 digested with NcoI and HindIII to give pHW22. The BspHI-EcoRI fragments of pHW15 and pHW16 was inserted into the NcoI and EcoRI sites of pET28b to give pHW39 and pHW28, respectively. By site-directed mutagenesis using the primers listed in Additional file 1, an NdeI site was introduced into pHW39 and pHW28. The NdeI-EcoRI fragment of this two new plasmids were inserted into the NdeI and EcoRI sites of pET28b to give pHW74 and pHW76. To increase FabZ expression, 24 codons that correspond to rare E. coli tRNA species were substituted with codons favored in $E$. coli by site-directed mutagenesis using the primers listed in Additional file 1 to give pHW74m. The NcoI-HindIII fragment of pHW74m was inserted into the NcoI and HindIII sites of pBAD24 to give pHW22m.

\section{Construction of an E. coli fabZ Deletion Strain}

A linear DNA fragments carrying a kan cassette was amplified from pKD13 by PCR [9,31] using primers, HZ1 and HZ2 listed in Additional file 1. These primers were homologous at the 3 ' end for priming sequences in pKD13 and contained 45-nucleotide extensions at the 5' end homologous to the E. coli fabZ sequence. The $1.4 \mathrm{~kb}$ PCR product was purified, treated with DpnI, and then introduced into a pHW22-containing derivative of DY330 a strain lysogenic for a defective prophage that contains the recombination genes under control of temperature-sensitive $c$ Irepressor [9]. The transformed cells were spread on LB plates containing ampicillin, kanamycin and arabinose. The E. coli fabZ deletion strain, HW7, was verified by PCR using primers P1, P2 plus HZ1, and HZ2.

\section{Analysis of phospholipid fatty acid compositions}

Cultures $(5 \mathrm{ml})$ were grown aerobically at different temperatures in RB medium overnight. The cells were then harvested and the phospholipids extracted as described previously [14]. The fatty acid compositions were analyzed by mass spectroscopy as described previously $[9,14]$. For analysis of radioactive fatty acids, $100 \mu \mathrm{l}$ of a culture grown overnight in LB medium was transferred into $5 \mathrm{ml}$ of RB medium supplemented with $0.1 \%$ cis-9, 10-methylenehexadecanoic acid (a cyclopropane fatty acid) plus $0.01 \%$ L-arabinose. After incubation of these cultures for $1 \mathrm{~h}, 5 \mu \mathrm{Ci}$ of sodium $\left[1-{ }^{14} \mathrm{C}\right]$ acetate was added and the culture allowed continuing growth for $4 \mathrm{~h}$. The phospholipids were then extracted as described above. The phospholipid acyl chains were converted to their methyl esters, which were separated by argentation thin-layer chromatography, and analyzed with autoradiography [12]

\section{Expression of plasmid-encoded proteins}

To assay expression of the products of C. acetobutylicium fabF1 and fabZ, pHW28 and pHW39 were introduced into E. coli strain BL21 (DE3), which encodes T7 RNA polymerase under the control of the IPTG-inducible lacUV5 promoter. The products of the cloned gene were selectively labeled with [35S]methionine as described [32]. The proteins were separated on a sodium dodecyl sulfate$12 \%$ polyacrylamide gel ( $\mathrm{pH} 8.8$ ). The destained gels were dried, and the labeled proteins were visualized by autoradiography [32]. The molecular mass markers (Bio-Rad, Richmond, Calif) were rabbit phosphorylase, bovine serum albumin, rabbit actin, bovine carbonic anhydrase, trypsin inhibitor and hen egg white lysozyme.

\section{Purification of FabFI and FabZ}

Plasmid pHW76 and pHW74m were introduced into strain BL21 (DE3), respectively, and the proteins were overexpressed and purified as described previously[20]. The enzymes were homogeneous as judged by sodium dodecyl sulfate-polyacrylamide gel electrophoresis. The E. coli FabD, FabH, FabG, FabA, FabZ, FabB, FabI and Vibrio harveyi AasS proteins were purified by their hexahisitidine tags described previously [18,20].

\section{Assay of FabFI and FabZ activity in vitro}

Fatty acid synthesis was reconstituted in vitro to assay FabF1 and FabZ activity using the purified enzymes that catalyze the fatty acid biosynthesis essentially. The assay utilized the AasS acyl-ACP synthetase from Vibrio harveyi [18] to generate 3-hydroxydecanoyl-ACP. The reaction mixtures to synthesize 3-hydroxydecanoyl-ACP contained $20 \mu \mathrm{M}$ ACP, $10 \mathrm{mM}$ ATP, $10 \mathrm{mM} \mathrm{MgSO}_{4}, 5 \mathrm{mM}$ DTT, 0.1 $\mathrm{M}$ sodium phosphate buffer (pH 7.0), $100 \mu \mathrm{M}$ 3-hydroxydecanoic acid (Sigma) and AasS $(0.2 \mu \mathrm{g})$ in a final volume of $50 \mu \mathrm{l}$. The mixtures were incubated at $37^{\circ} \mathrm{C}$ for 1 h. To assay C. acetobutylicium FabF1, the following incubation $1 \mu \mathrm{g}$ each of E. coli FabD, FabG and FabA, $100 \mu \mathrm{M}$ NADPH, $100 \mu \mathrm{M}$ NADH, $100 \mu \mathrm{M}$ malonyl-CoA, and $1 \mu \mathrm{g}$ of either E. coli FabB or C. acetobutylicium FabF1 was added. To assay C. acetobutylicium FabZ, the following incubation contained $1 \mu \mathrm{g}$ each of $E$. coli FabD, FabG and FabB, $100 \mu \mathrm{M}$ NADPH, $100 \mu \mathrm{M}$ NADH, $100 \mu \mathrm{M}$ malonylCoA, and $1 \mu \mathrm{g}$ of E. coli FabA or C. acetobutylicium FabZ was added. The resulting mixture was incubated for an additional $1 \mathrm{~h}$ and the reaction products were analyzed by conformationally sensitive gel electrophoresis on $20 \%$ polyacrylamide gels containing $2.5 \mathrm{M}$ urea $[20,24]$. The gel was stained with Coomassie Brilliant Blue R250.

\section{Authors' contributions}

LZ cloned Clostridium acetobutylicium fabFs genes, constructed several $f a b F$ expression vectors and did complementation experiments with $f a b F s$ expression vectors. JC 
cloned Clostridium acetobutylicium fabZ gene and made E. coli fabZ mutant. BL changed codons that correspond to rare E. coli tRNA species in C. acetobutylicium fabZ to codons favored in E. coli by site-directed mutagenesis. SF carried out biochemical studies on FabF and FabZ of $C$. acetobutylicium in vitro. JL performed expression experiments and purified FabF and FabZ proteins. SW helped to design the PCR primers. JEC participated in the design of the study and helped to draft the manuscript. HW conceived of the study, and participated in its design and coordination and helped to draft the manuscript. All authors read and approved the final manuscript.

\section{Additional material}

\section{Additional file 1}

Bacterial strains, plasmids and oligonucleotides used in this work. The data provided bacteria strains, plasmids and oligonucleotides used in this work.

Click here for file

[http://www.biomedcentral.com/content/supplementary/14712180-9-119-S1.pdf]

\section{Acknowledgements}

This work was supported by the President Foundation of South China Agricultural University and NIH grant AlI5650. We are grateful to Professor Hiroshi Kobayashi (Graduate School of Pharmaceutical Sciences, Chiba University Japan) for critical reading.

\section{References}

I. Cronan JE: Bacterial membrane lipids: where do we stand? Annu Rev Microbiol 2003, 57:203-224.

2. Mansilla MC, de Mendoza D: The Bacillus subtilis desaturase: a model to understand phospholipid modification and temperature sensing. Archives of microbiology 2005, I 83(4):229-235.

3. Bloch K, Baronowsky P, Goldfine H, Lennarz WJ, Light R, Norris AT, Scheuerbrandt G: Biosynthesis and metabolism of unsaturated fatty acids. Fed Proc 1961, 20:921-927.

4. Scheuerbrandt G, Goldfine H, Baronowsky PE, Bloch K: A novel mechanism for the biosynthesis of unsaturated fatty acids. I Biol Chem 1961, 236:PC70-PC7I.

5. Bloch K: Beta-Hydroxythioester dehydrase. In The enzymes Volume 5. 3rd edition. Edited by: Boyer PD. New York: Academic press; 1971:44|-464.

6. Campbell JW, Cronan JE Jr: Bacterial fatty acid biosynthesis: targets for antibacterial drug discovery. Annu Rev Microbiol 200I, 55:305-332.

7. Lu YJ, Zhang YM, Rock CO: Product diversity and regulation of type II fatty acid synthases. Biochem Cell Biol 2004, 82(I): |45-155.

8. Marrakchi H, Zhang YM, Rock CO: Mechanistic diversity and regulation of Type II fatty acid synthesis. Biochem Soc Trans 2002, 30(Pt 6): 1050-1055.

9. Wang H, Cronan JE: Functional replacement of the FabA and FabB proteins of Escherichia coli fatty acid synthesis by Enterococcus faecalis FabZ and FabF homologues. I Biol Chem 2004, 279(33):34489-34495.

10. Nolling J, Breton G, Omelchenko MV, Makarova KS, Zeng Q, Gibson R, Lee HM, Dubois J, Qiu D, Hitti J, et al:: Genome sequence and comparative analysis of the solvent-producing bacterium Clostridium acetobutylicum. 2001 , I 83( ( 6):4823-4838.

II. Garwin JL, Klages AL, Cronan JE Jr: Beta-ketoacyl-acyl carrier protein synthase II of Escherichia coli. Evidence for function in the thermal regulation of fatty acid synthesis. I Biol Chem 1980, 255(8):3263-3265.

12. Ulrich AK, de Mendoza D, Garwin JL, Cronan JE Jr: Genetic and biochemical analyses of Escherichia coli mutants altered in the temperature-dependent regulation of membrane lipid composition. J Bacteriol I983, I 54(I):22 I-230.

13. de Mendoza $D$, Cronan JE Jr: Thermal regulation of membrane lipid fluidity in bacteria. Trends BiochemSci 1983, 8:49-52.

14. Wang H, Cronan JE: Haemophilus influenzae Rd lacks a stringently conserved fatty acid biosynthetic enzyme and thermal control of membrane lipid composition. J Bacteriol 2003, 185(16):4930-4937.

15. Gerdes SY, Scholle MD, Campbell JW, Balazsi G, Ravasz E, Daugherty MD, Somera AL, Kyrpides NC, Anderson I, Gelfand MS, et al.: Experimental determination and system level analysis of essential genes in Escherichia coli MGI655. J Bacteriol 2003, 185( I 9):5673-5684.

16. Baba T, Ara T, Hasegawa M, Takai Y, Okumura Y, Baba M, Datsenko KA, Tomita M, Wanner BL, Mori H: Construction of Escherichia coli K-I 2 in-frame, single-gene knockout mutants: the Keio collection. Mol Syst Biol 2006, 2:0008.

17. Yu D, Ellis HM, Lee EC, Jenkins NA, Copeland NG, Court DL: An efficient recombination system for chromosome engineering in Escherichia coli. Proc Natl Acad Sci USA 2000, 97( I I ):5978-5983.

18. Jiang $\mathrm{Y}$, Chan $\mathrm{CH}$, Cronan JE: The soluble acyl-acyl carrier protein synthetase of Vibrio harveyi B392 is a member of the medium chain acyl-CoA synthetase family. Biochemistry 2006 , 45(33): 10008-10019.

19. Guerra DJ, Browse JA: Escherichia coli beta-hydroxydecanoyl thioester dehydrase reacts with native $\mathrm{CI} O \mathrm{acyl}$-acyl-carrier proteins of plant and bacterial origin. Arch Biochem Biophys 1990, 280(2):336-345.

20. Heath RJ, Rock CO: Roles of the FabA and FabZ beta-hydroxyacyl-acyl carrier protein dehydratases in Escherichia coli fatty acid biosynthesis. J Biol Chem I 996, 27 I (44):27795-2780 I.

21. Johnston NC, Goldfine H: Lipid composition in the classification of the butyric acid-producing clostridia. J Gen Microbiol I983, I 29(4): |075-108|.

22. Goldfine $\mathrm{H}$, Bloch $\mathrm{K}$ : On the origin of unsaturated fatty acids in clostridia. J Biol Chem I96I, 236:2596-260I.

23. Kimber MS, Martin F, Lu Y, Houston S, Vedadi M, Dharamsi A, Fiebig KM, Schmid M, Rock CO: The structure of (3R)-hydroxyacylacyl carrier protein dehydratase (FabZ) from Pseudomonas aeruginosa. I Biol Chem 2004, 279(50):52593-52602.

24. Lu YJ, White SW, Rock CO: Domain swapping between Enterococcus faecalis FabN and FabZ proteins localizes the structural determinants for isomerase activity. I Biol Chem 2005, 280(34):30342-30348.

25. Clark DP, DeMendoza D, Polacco ML, Cronan JE Jr: Beta-hydroxydecanoyl thio ester dehydrase does not catalyze a rate-limiting step in Escherichia coli unsaturated fatty acid synthesis. Biochemistry 1983, 22(25):5897-5902.

26. Cornillot E, Nair RV, Papoutsakis ET, Soucaille P: The genes for butanol and acetone formation in Clostridium acetobutylicum ATCC 824 reside on a large plasmid whose loss leads to degeneration of the strain. J Bacteriol 1997, I 79( I 7):5442-5447.

27. Morgan-Kiss RM, Cronan JE: The Lactococcus lactis FabF fatty acid synthetic enzyme can functionally replace both the FabB and FabF proteins of Escherichia coli and the FabH protein of Lactococcus lactis. Archives of microbiology 2008, I 90(4):427-437.

28. Takeshita S, Sato M, Toba M, Masahashi W, Hashimoto-Gotoh T: High-copy-number and low-copy-number plasmid vectors for lacZ alpha-complementation and chloramphenicol- or kanamycin-resistance selection. Gene 1987, 6 I ( I):63-74.

29. Bartolome B, Jubete $Y$, Martinez E, de la Cruz F: Construction and properties of a family of pACYC I 84-derived cloning vectors compatible with pBR322 and its derivatives. Gene 1991, I 02(I):75-78

30. Guzman LM, Belin D, Carson MJ, Beckwith J: Tight regulation, modulation, and high-level expression by vectors containing the arabinose PBAD promoter. J Bacteriol 1995 , I77(14):4I2I-4I30.

31. Datsenko KA, Wanner BL: One-step inactivation of chromosomal genes in Escherichia coli K-I 2 using PCR products. Proc Natl Acad Sci USA 2000, 97( I 2):6640-6645 
32. Choi-Rhee E, Cronan JE: The biotin carboxylase-biotin carboxyl carrier protein complex of Escherichia coli acetyl-CoA carboxylase. J Biol Chem 2003, 278(33):30806-308I2.

33. Campbell JW, Cronan JE Jr: Escherichia coli FadR positively regulates transcription of the fabB fatty acid biosynthetic gene. J Bacteriol 200I, I 83(20):5982-5990.

34. Schairer HU, Overath $\mathrm{P}$ : Lipids containing trans-unsaturated fatty acids change the temperature characteristic of thiomethylgalactoside accumulation in Escherichia coli. J Mol Biol 1969, 44(I):209-2।4.

35. Magnuson K, Carey MR, Cronan JE Jr: The putative fabJ gene of Escherichia coli fatty acid synthesis is the fabF gene. J Bacteriol 1995, I77(12):3593-3595.

Publish with Bio Med Central and every scientist can read your work free of charge

"BioMed Central will be the most significant development for disseminating the results of biomedical research in our lifetime. "

Sir Paul Nurse, Cancer Research UK

Your research papers will be:

- available free of charge to the entire biomedical community

- peer reviewed and published immediately upon acceptance

- cited in PubMed and archived on PubMed Central

- yours - you keep the copyright

Submit your manuscript here:

http://www.biomedcentral.com/info/publishing_adv.asp
BioMedcentral 